\title{
Human CYP7A1 deficiency: progress and enigmas
}

Commentary

\section{Anne Beigneux, ${ }^{1}$ Alan F. Hofmann, ${ }^{2}$ and Stephen G. Young ${ }^{1}$ \\ ${ }^{1}$ Gladstone Institute of Cardiovascular Disease, Cardiovascular Research Institute, and Department of Medicine, University of California, San Francisco, San Francisco, California, USA \\ ${ }^{2}$ Department of Medicine, University of California, San Diego, La Jolla, California, USA}

Address correspondence to: Stephen G. Young, Gladstone Institute of Cardiovascular Disease, 365 Vermont Street, San Francisco, California 94103, USA. Phone: (415) 826-7500; Fax: (415) 285-5632; E-mail: syoung@gladstone.ucsf.edu.

J. Clin. Invest. 110:29-31 (2002). doi:10.1172/JCI200216076.

Studies of human mutations have paved the way toward understanding elevated plasma cholesterol levels, both by exposing the mechanisms of lipid transport and by focusing hypotheses for further studies $(1,2)$. In this issue, Pullinger et al. (3) studied a kindred with hypercholesterolemia and identified a mutation in CYP7A1, which encodes cholesterol $7 \alpha$-hydroxylase, the first and rate-limiting step in the classical bile acid synthetic pathway (4). As has been the case before, this new mutation has simultaneously shed light on mechanisms of lipid metabolism and raised a welter of issues for future investigation.

The study by Pullinger et al. (3) must have been supremely gratifying for the authors: They started with an imaginative hypothesis about hypercholesterolemia - one rooted in simple clinical findings - and, after some serious screening efforts, hit pay dirt. They hypothesized that CYP7A1 deficiency would reduce the conversion of cholesterol to bile acids, resulting in elevated liver cholesterol levels, downregulated LDL receptors, and hypercholesterolemia. They further hypothesized that the increased hepatic cholesterol levels would render these patients resistant to the hypocholesterolemic effect of statins and that CYP7A1 deficiency would cause premature gallstone disease, a result of reduced bile acid secretion rates. By screening appropriate patients from their lipid clinic, they identified two brothers, both with premature gallstone disease and statinresistant hypercholesterolemia, who were homozygous for a frameshift mutation in CYP7A1 that abolished enzyme activity. A third homozygote also had hypercholesterolemia, and six heterozygotes had higher cholesterol levels than unaffected family members, leading the authors to conclude that CYP7A1 deficiency in humans causes hypercholesterolemia. This conclusion is consistent with population studies that have shown an association between plasma cholesterol levels and polymorphisms at the CYP7A1 locus $(5,6)$. a

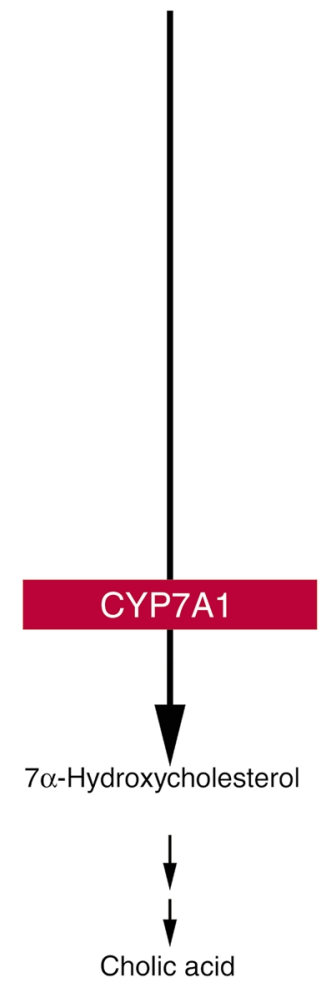

b Cholesterol

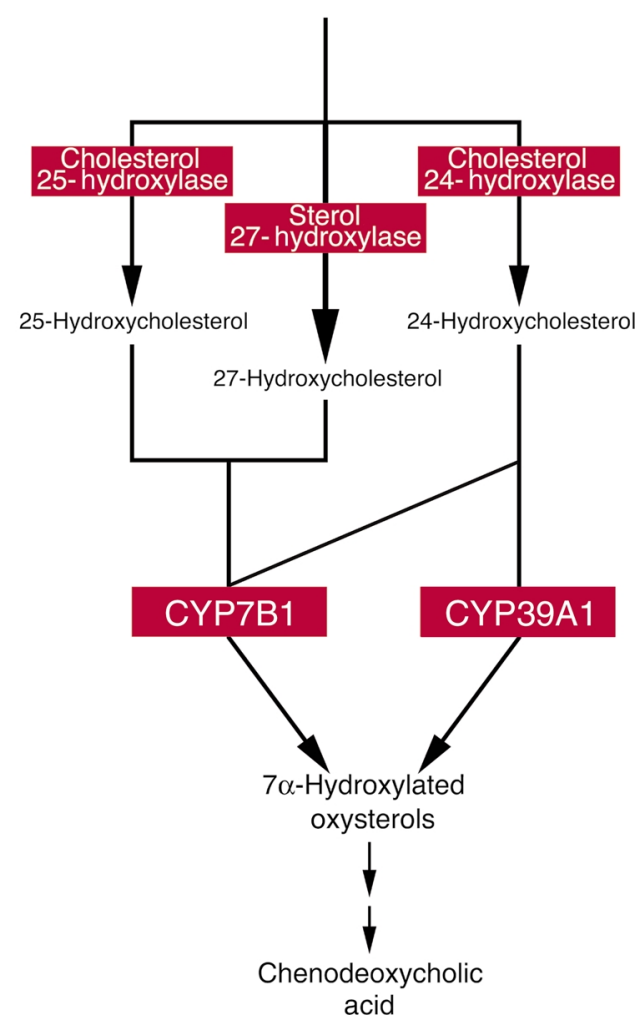

Figure 1

(a) The classical pathway for bile acid synthesis begins with CYP7A1, which converts cholesterol into $7 \alpha$-hydroxycholesterol. This pathway mainly produces cholic acid in humans. (b) Alternate pathways for bile acid synthesis. Cholesterol is first converted into oxysterols by one of three different enzymes: sterol 27-hydroxylase (CYP27), expressed in multiple tissues including liver; cholesterol 25-hydroxylase, present at low levels in multiple tissues including heart, lung, and kidney; and cholesterol 24-hydroxylase (CYP46), expressed predominantly in the brain. Oxysterols are transported through the bloodstream to the liver, where they are $7 \alpha$-hydroxylated by oxysterol $7 \alpha$-hydroxylase (CYP7B1) in the case of 25- and 27-hydroxycholesterol and by CYP39A1 in the case of 24-hydroxycholesterol. Alternate pathways preferentially produce chenodeoxycholic acid in humans. 
ity of ascertainment bias must be considered, particularly since their kindred was not particularly large and since the conclusions were based on a limited number of lipid measurements. More kindreds with CYP7A1 mutations will be needed if the phenotype is to be defined conclusively.

Assuming that the hypercholesterolemic phenotype is upheld by future studies, it will be crucial to explore mechanisms. Their hypothesis holds that CYP7A1-deficient subjects have elevated liver cholesterol levels leading to reduced LDL receptor activity and retarded removal of LDL from the bloodstream. This hypothesis must be tested. The VLDL and LDL turnover rates in CYP7A1deficient subjects should be measured and compared with those in both normal controls and subjects with the heterozygous form of familial hypercholesterolemia.

\section{CYP7A1 and triglycerides}

A surprising and unexplained finding in the current study was the observation that two of the three homozygotes were hypertriglyceridemic. For years, lipidologists have observed hypertriglyceridemia in patients on bile acid sequestrants, where bile acid synthesis rates are high $(7,8)$. It seems curious that low levels of bile acid synthesis, as in CYP7A1 deficiency, should also be associated with hypertriglyceridemia. Several conceivable mechanisms for hypertriglyceridemia could be proposed. Low levels of bile acids might downregulate farnesoid $\mathrm{X}$ receptor-responsive gene products, including apoC-II, an activator of lipoprotein lipase, the enzyme that removes triglycerides from the plasma (9). Alternatively, it is possible that elevated oxysterol levels in the liver might induce liver $\mathrm{X}$ receptor-responsive genes, leading to increased SREBP-1C expression and increased triglyceride synthesis $(10,11)$. Consistent with the latter model, Pullinger and colleagues (3) found higher levels of an oxysterolproducing enzyme (sterol-27 hydroxylase) in a liver biopsy from one of their homozygotes. However, it is not clear that elevated levels of the enzyme would necessarily cause higher steadystate levels of oxysterols in hepatocytes, as the oxysterols can be converted rapidly to bile acids via an alternate pathway not involving CYP7A1.

\section{Qualitative and quantitative effects on bile acids}

Pullinger et al. (3) report a 94\% reduction in bile acids excretion in one of their homozygotes. In a healthy person, cholic acid synthesis exceeds synthesis of chenodeoxycholic acid by a factor of two. In their patient, fecal bile acid analysis showed that the major bile acid formed was chenodeoxycholic acid. The fact that CYP7A1 deficiency did not abolish bile acid formation is not surprising, since there are several pathways, differing in their initial steps, for conversion of cholesterol into bile acids (Figure 1) $(12,13)$. The classical neutral pathway involves CYP7A1, which directly converts cholesterol into $7 \alpha$-hydroxycholesterol (Figure 1a). In the alternate acidic pathway, cholesterol is first converted by sterol hydroxylases into oxysterols, the major one being 27-hydroxycholesterol. These oxysterols then undergo $7 \alpha$-hydroxylation by an oxysterol $7 \alpha$-hydroxylase, either CYP7B1 or CYP39A1 (Figure 1b). Subsequent enzymatic steps lead to the preferential conversion of $7 \alpha$-hydroxycholesterol into cholic acid and the preferential conversion of $7 \alpha$-hydroxy-oxysterols into chenodeoxycholic acid.

The shift in primary bile acid synthesis toward chenodeoxycholic acid is entirely consistent with decreased CYP7A1 activity and compensation by the acidic pathway. Of note, a low bile acid synthetic rate and preferential formation of chenodeoxycholic acid were observed previously in two hypercholesterolemic patients whose bile acid synthesis profile was characterized by Einarsson et al. (14) a quarter century ago. We wonder whether those patients might have had CYP7A1 mutations.

The $94 \%$ reduction in fecal bile acid excretion (equivalent at steady state to hepatic bile acid synthesis) was observed in a single 24-hour stool collection in a single subject. A longer stool collection would seem essential for quantifying accurately the decreased rate of bile acid synthesis. Future studies should be directed at clarifying this issue as well as determining the rate of bile acid secretion, which can be measured by duodenal intubation and isotopic tracer studies. Better characterization of bile acid biosynthesis in human CYP7A1-deficient patients is particularly important given a distinct lack of evidence for cholesterol or vitamin malabsorption in those individuals. Given that adequate intraluminal concentrations of bile acids are essential for the absorption of cholesterol and fat-soluble vitamins, it seems possible that increased intestinal reclamation of bile acids in CYP7A1-deficient patients may have compensated for their decrease in bile acid biosynthesis. In the guinea pig, low concentrations of bile acids within the lumen of the intestine upregulate the ileal bile acid transport system (15).

\section{Differences in CYP7A1-deficient mice and humans}

The normal cholesterol absorption and normal vitamin $E$ levels in humans with CYP7A1 deficiency contrasts with findings in Cyp7a1-deficient mice, where the bile acid pool size is reduced by $80 \%$. In those mice, cholesterol absorption is almost abolished (16), and tissue vitamin E levels are markedly reduced (17). Pullinger et al. (3) also found triglyceride malabsorption in one of the homozygotes a very unexpected finding given that triglyceride absorption is normal in the Cyp7a1-deficient mice (16).

A single liver biopsy in one subject suggested that liver cholesterol levels might be increased in CYP7A1-deficient humans. That observation, although in accordance with the authors' hypothesis, would not have been predicted from animal models (16). In chow-fed Cyp7a1-deficient mice, liver cholesterol levels are not increased, and cholesterol synthesis rates actually increase, likely secondary to reduced absorption of cholesterol in the intestine (16). When additional humans with CYP7A1 deficiency are identified - if liver biopsies are possible - it will be very interesting to determine if elevated liver cholesterol levels are a consistent feature of CYP7A1 deficiency. It will also be desirable to examine the expression level of the LDL receptor and other gene products regulated by SREBPs.

Additional mysteries are apparent when one compares Cyp7a1-deficient mice and the CYP7A1-deficient humans. The humans were hyperlipidemic, but the mice were not (17), at least when originally characterized (although see refs. 18-20 for evidence of hypercholesterolemia in some genetic backgrounds). Also, in the case of the mice, Cyp7a1 deficiency abolished cho- 
lesterol $7 \alpha$-hydroxylase activity in the liver, but in the humans, the liver biopsy study suggested that cholesterol $7 \alpha-$ hydroxylase activity was reduced by only $\sim 70 \%$. This difference raises the possibility that some other enzyme, perhaps an oxysterol $7 \alpha$-hydroxylase, can hydroxylate the C-7 position of cholesterol in humans. Finally, increased sterol-27 hydroxylase levels in a liver biopsy from one of the CYP7A1deficient patients suggest that the oxysterol pathway is upregulated in humans, a response that was not observed in Cyp7a1-deficient mice (13).

Cyp7a1 expression is critical for mice in the postnatal period because Cyp7b1 expression is not turned on until 3 weeks of age. Most Cyp7a1deficient mice die before weaning unless they are supplemented with cholic acid and fat-soluble vitamins (21). In contrast, as noted above, there was no indication that the CYP7A1deficient patients had significant fat malabsorption or fat-soluble vitamin deficiencies as children. Thus, the activity of alternate bile acid synthesis pathways together with intestinal conservation of bile acids was apparently sufficient to prevent overt lipid malabsorption.

Humans and mice also exhibit different phenotypes for mutations in other bile acid metabolism-related proteins. A human infant lacking CYP7B1, who died with severe biliary cholestasis, has been described (22). In contrast, Cyp7b1-deficient mice appear perfectly healthy (23). Mice deficient in the canalicular bile salt export pump have mild cholestasis (24), whereas humans with this defect develop progressive familial cholestasis, a merciless pediatric disease causing early cirrhosis (25). Loss of sterol 27-hydroxylase in humans causes a striking deficiency of bile acids, cerebrotendinous xanthomatosis, and an accumulation of cholestanol in tissues (26-28). Mice with the same enzyme defect have a bile acid synthesis defect but do not develop cerebrotendinous xanthomatosis. Instead, they manifest elevated triglyceride levels in liver and plasma (29). Thus, when it comes to disorders of bile acid and cholesterol metabolism, clinical investigators would be wise to study both mice and humans.

1. Brown, M.S., and Goldstein, J.L. 1986. A receptormediated pathway for cholesterol homeostasis Science. 232:34-47.

2. Vega, G.L., and Grundy, S.M. 1986. In vivo evidence for reduced binding of low density lipoproteins to receptors as a cause of primary moderate hypercholesterolemia. J. Clin. Invest. 78:1410-1414.

3. Pullinger, C.R., et al. Human cholesterol $7 \alpha$-hydroxlyase (CYP7A1) deficiency has a hypercholesterolemic phenotype. J. Clin. Invest 110:109-117. doi:10.1172/JCI200215387.

4. Shefer, S., Hauser, S., Bekersky, I., and Mosbach, E.H. 1970. Biochemical site of regulation of bile acid biosynthesis in the rat. J. Lipid Res. 11:404-411.

5. Wang, J., et al. 1998. Linkage between cholesterol $7 \alpha$-hydroxylase and high plasma low-density lipoprotein cholesterol concentrations. J. Clin. Invest. 101:1283-1291.

6. Couture, P., et al. 1999. Association of the A-204C polymorphism in the cholesterol $7 \alpha$-hydroxlase gene with variation in plasma low density lipoprotein cholesterol levels in the Framingham Offspring Study. J. Lipid Res. 40:1883-1889.

7. Crouse, J.R., III. 1987. Hypertriglyceridemia: a contraindication to the use of bile acid binding resins. Am. J. Med. 83:243-248.

8. Beil, U., Crouse, J.R., Einarsson, K., and Grundy, S.M. 1982. Effects of interruption of the enterohepatic circulation of bile acids on the transport of very low density-lipoprotein triglycerides. Metabolism. 31:438-444.

9. Kast, H.R., et al. 2001. Farnesoid X-activated receptor induces apolipoprotein C-II transcription: a molecular mechanism linking plasma triglyceride levels to bile acids. Mol. Endocrinol. 15: $1720-1728$

10. Repa, J.J., et al. 2000. Regulation of mouse sterol regulatory element-binding protein-1c gene (SREBP-1c) by oxysterol receptors, LXRalpha and LXRbeta. Genes Dev. 14:2819-2830.

11. Fu, X., et al. 2001. 27-hydroxycholesterol is an endogenous ligand for liver $\mathrm{X}$ receptor in cholesterol-loaded cells. J. Biol. Chem. 276:38378-38387.

12. Russell, D.W. 2000. Oxysterol biosynthetic enzymes. Biochim. Biophys. Acta. 1529:126-135.

13. Schwarz, M., Russell, D.W., Dietschy, J.M., and Turley, S.D. 2001. Alternate pathways of bile acid synthesis in the cholesterol $7 \alpha$-hydroxylase knockout mouse are not upregulated by either cholesterol or cholestyramine feeding. J. Lipid Res. 42:1594-1603

14. Einarsson, K., Hellstrom, K., and Kallner, M. 1974. Bile acid kinetics in relation to sex, serum lipids, body weights, and gallbladder disease in patients with various types of hyperlipoproteinemia. J. Clin. Invest. 54:1301-1311.

15. Lillienau, J., et al. 1993. Negative feedback regulation of the ileal bile acid transport system in rodents. Gastroenterology. 104:38-46.

16. Schwarz, M., Russell, D.W., Dietschy, J.M., and Turley, S.D. 1998. Marked reduction in bile acid synthesis in cholesterol $7 \alpha$-hydroxylase-deficient mice does not lead to diminished tissue cholesterol turnover or to hypercholesterolemia. J. Lipid Res. 39:1833-1843.

17. Schwarz, M., et al. 1996. Disruption of cholesterol $7 \alpha$-hydroxylase gene in mice. II. Bile acid deficiency is overcome by induction of oxysterol $7 \alpha$ hydroxylase. J. Biol. Chem. 271:18024-18031.

18. Shefer, S., Erickson, S.K., Lear, S.R., Batta, A.K. and Salen, G. 1999. Upregulation of hepatic cholesterol 27-hydroxylase (Cyp27) activity and normal levels of hepatic cholesterol synthesis in cholesterol $7 \alpha$-hydroxylase (Cyp7a1) gene knockout mice. Gastroenterology. 118:1168.

19. Erickson, S.K., and Lear, S.R. 1999. Effect of cholesterol $7 \alpha$-hydroxylase (cyp7a) gene knockout on lipid homeostasis. Circulation. 100:I-686 (Abstr.)

20. Erickson, S.K. 1999. Cholesterol $7 \alpha$-hydroxylase (Cyp7a) gene knockout results in hypercholesterolemia, gender-specific alteration in bile acid pool size and composition and in gender-specific hyperinsulinemia. Hepatology. 30:630.

21. Ishibashi, S., Schwarz, M., Frykman, P.K., Herz, J., and Russell, D.W. 1996. Disruption of cholesterol $7 \alpha$-hydroxylase gene in mice. I. Postnatal lethality reversed by bile acid and vitamin supplementation. J. Biol. Chem. 271:18017-18023.

22. Setchell, K.D.R., et al. 1998. Identification of a new inborn error in bile acid synthesis: mutation of the oxysterol $7 \alpha$-hydroxylase gene causes severe neonatal liver disease. J. Clin. Invest. 102:1690-1703.

23. Li-Hawkins, J., Lund, E.G., Turley, S.D., and Russell, D.W. 2000. Disruption of the oxysterol $7 \alpha$ hydroxylase gene in mice. J. Biol. Chem. 275:16536-16542.

24. Wang, R., et al. 2001. Targeted inactivation of sister of P-glycoprotein gene ( $s p g p)$ in mice results in nonprogressive but persistent intrahepatic cholestasis. Proc. Natl. Acad. Sci. USA. 98:2011-2016

25. Jansen, P.L., et al. 1999. Hepatocanalicular bile salt export pump deficiency in patients with progres sive familial intrahepatic cholestasis. Gastroenterology. 117:1370-1379.

26. Salen, G. 1971. Cholestanol deposition in cerebrotendinous xanthomatosis. A possible mechanism. Ann. Intern. Med. 75:843-851.

27. Salen, G., and Grundy, S.M. 1973. The metabolism of cholestanol, cholesterol, and bile acids in cerebrotendinous xanthomatosis. J. Clin. Invest. 52:2822-2835.

28. Salen, G., et al. 1985. Biosynthesis of bile acids in cerebrotendinous xanthomatosis. Relationship of bile acid pool sizes and synthesis rates to hydroxylations at C-12, C-25, and C-26. J. Clin. Invest. 76:744-751.

29. Repa, J.J., et al. 2000. Disruption of the sterol 27hydroxylase gene in mice results in hepatomegaly and hypertriglyceridemia. Reversal by cholic acid feeding. J. Biol. Chem. 275:39685-39692. 